
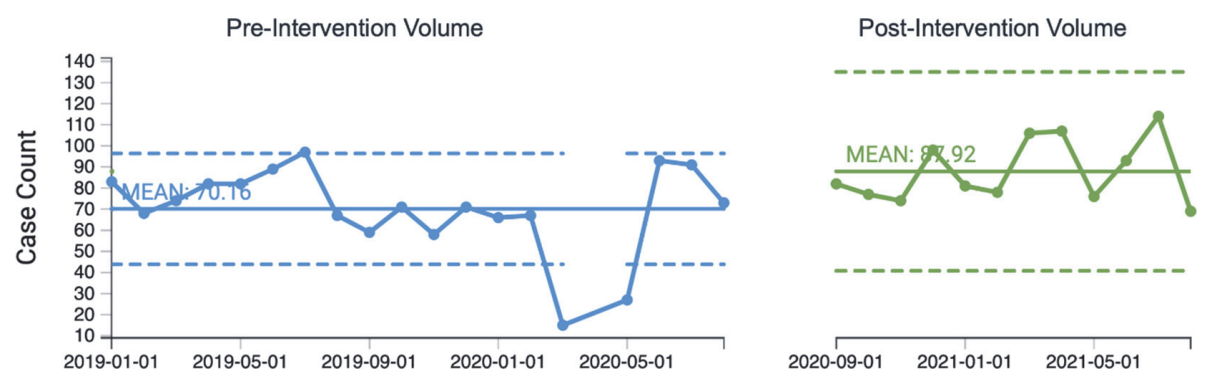

Abstract 5 Figure 5 Team increases monthly volume by $25 \%$ - Improvement demonstrated by centerline shift from 70 to 88 cases a month

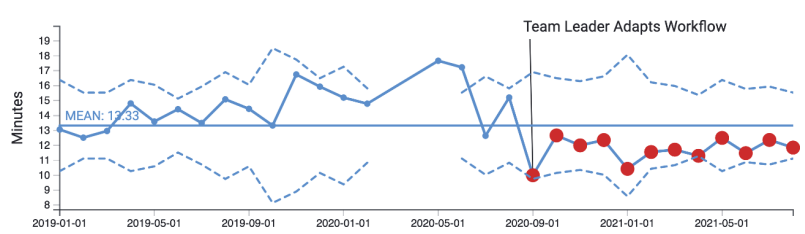

Abstract 5 Figure 3 Team leader adapts personal workflow improvement demonstrated by special cause variation (highlighted)

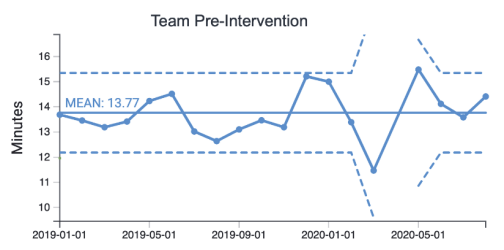

Team Post-Intervention

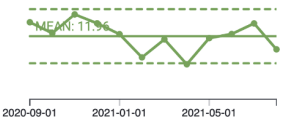

Abstract 5 Figure 4 Team adapts workflow - improvement demonstrated by centerline shift from 13.8 to 11.9 minutes

identified, including specific tasks performed during the surgical prep time, solutions used for prepping, task sequencing, and interdisciplinary collaboration. The team leader adapted their personal workflow in September 2021 and demonstrated a reduction in surgical prep times (figure 4). Team performance also improved (figure 5), surgical prep times reduced to 11.9 minutes. The team performed at least one additional surgery per day, resulting in a $25 \%$ increase in case volume (figure 5), from 70 cases per month to 88 cases per month

Conclusions Resource-neutral, data driven small system improvements, resulted in a $25 \%$ increase in case volumes post intervention, creating $>\$ 2.5 \mathrm{M}$ additional revenue.

\section{TRANSFORMING A NATIONAL LABORATORY NETWORK TO MEET COVID-19 TESTING DEMAND}

John Boulton, lain Roberts, Kelly Ward. Public Health Wales NHS Trust

\subsection{6/bmjoq-2021-IHI.6}

Background Public Health Wales NHS Trust (PHW) is responsible for Microbiology laboratory services that support NHS Wales. The testing capacity within the laboratories was increased rapidly. There was a requirement to improve laboratory turnaround time for samples to enable results to be provided to individuals as quickly as possible.

As laboratory capacity increased, turnaround times did not improve. The following issues were identified:

- Variation in turnaround times for different populations.
- A lack of understanding of daily demand for tests

- Lack of standard processes for handling samples upon arrival in the laboratories

Objectives The aim was that within 3 months, $80 \%$ of all samples would be turned around within 12 hours of receipt in the laboratory. A stretch goal of achieving $95 \%$ by the summer. The work consisted of:

- Engagement with all laboratories

- Mapping sample flows

- Development of dashboards to understand flow, demand and activity

- Mapping of in-laboratory processes

- Modelling sample flows

Methods Using a Lean methodology, the following were implemented:

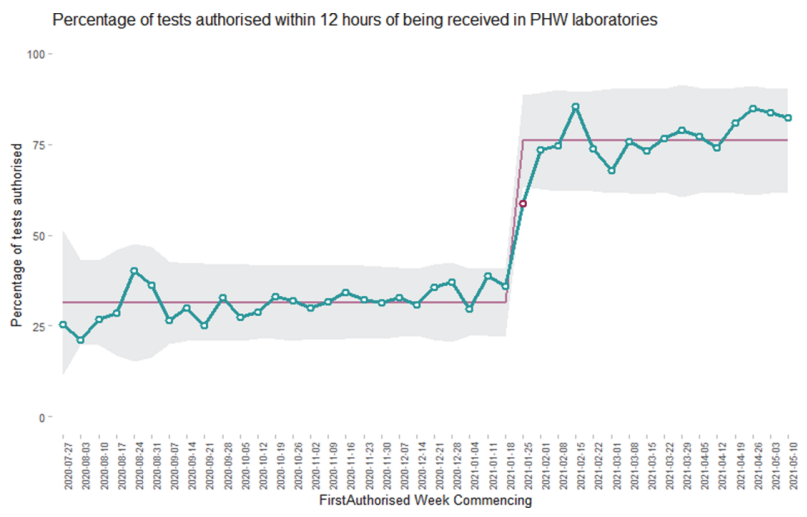

Abstract 6 Figure 1

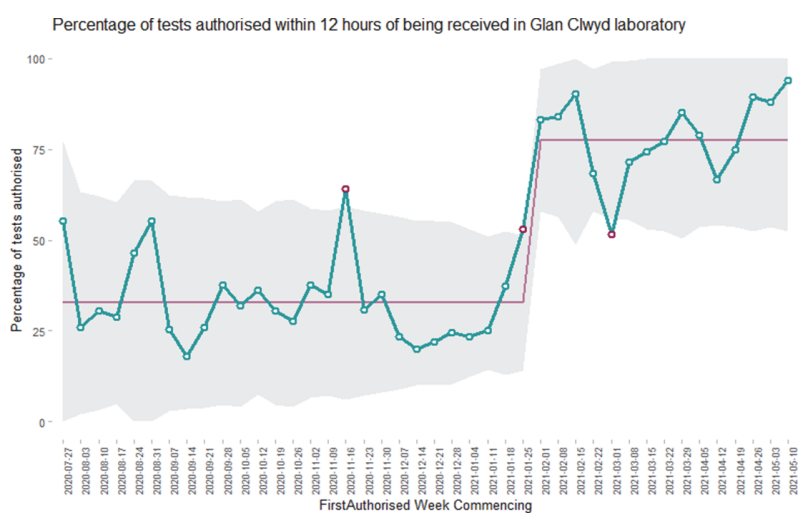

Abstract 6 Figure 2 


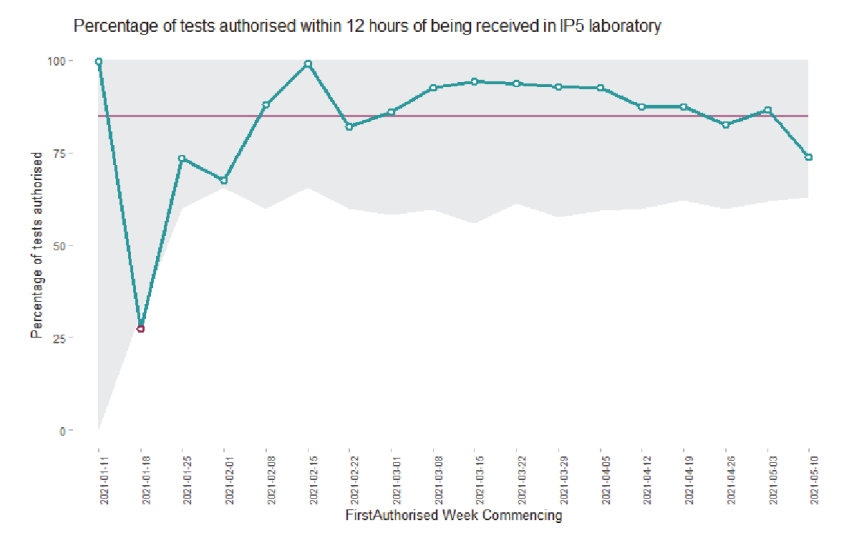

Abstract 6 Figure 3

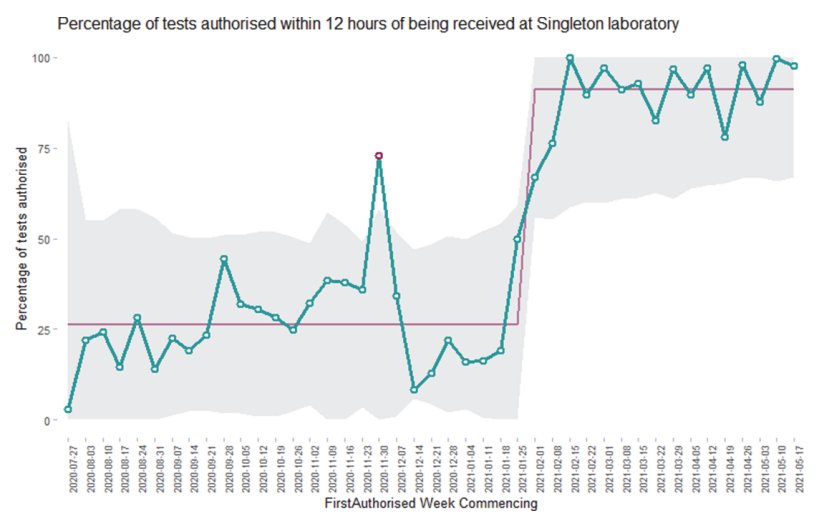

Abstract 6 Figure 4

- Flow of all asymptomatic community samples to a dedicated high volume laboratory

- Standard process for receipt of samples, including prioritisation

- Improved flow of samples around laboratories

- Daily meetings to discuss on-going issues and an opportunity to share good practice

- Laboratory process redesign

Results Improvement work commenced in January 2021. As a consequence, times improved across all laboratories (figures 14). Median turnaround time reduced from over 24 hours to 6 hours. This has been sustained. $80 \%$ of samples are now turned around within 12 hours.
Conclusions The need for rapid turnaround of Covid-19 samples is essential. As a consequence of a national improvement program $80 \%$ of all samples have a turnaround time of 12 hours of less.

\section{ANGEL OF LOVE: THE STORY OF FILM-MAKING IN AN ACUTE DIALYSIS UNIT IN A TIME OF COVID-19}

${ }^{1}$ Nicola Abraham, ${ }^{2}$ Jo James. ${ }^{1}$ Royal Central School of Speech and drama; ${ }^{2}$ Imperial College Healthcare NHS Trust

\subsection{6/bmjoq-2021-IHI.7}

Background Auchi dialysis unit supports patients who have complex needs and require more intensive care. During the pandemic, many of the patients had to isolate from family for months and were frightened about having to come into hospital. They became demoralized and disengaged. This project connected applied theatre students from Royal Central School of Speech and Drama with the patients.

Objectives The aim was to provide social connection, stimulation and joy by creating a film through a collaboration with patients and students based on the creative output of the patients.

Methods Multiple short PDSA cycles were undertaken to determine the best way to connect digitally and deliver the project. Staff identified patients that would benefit from the project. Patients then participated in interactive narrative workshops twice a week for 6 weeks via zoom (figure 3 and 4). These were facilitated by the students, supported in person by the project team (figure 1).

Results Results were measured through feedback from the participants and staff and also by the quality of the film produced. Qualitative data from staff observations and patient feedback has revealed the positive impact of the project on patient wellbeing. Project team observations and patient feedback revealed 'focus' on collaborative film making changed the mood of the patients during workshops (figure 2).

Conclusions Using Quality improvement methodology in our collaborative film making enabled us to continually improve the experience and inclusion of patients in a creative project. This allowed us to overcome the limitations of PPE and internet connectivity and devise a transmedia approach to maximise the opportunities for the patients to be creative (figure $5)$.

\begin{tabular}{|l|c|}
\hline Cycle Description & Number \\
\hline Internet connectivity session & 4 \\
\hline $\begin{array}{l}\text { Communication test - ways to help patients understand facilitator's instructions } \\
\text { with masks and face shields. }\end{array}$ & 2 \\
\hline $\begin{array}{l}\text { Testing transmedia approach - using simultaneous What's App to provide } \\
\text { facilitator clarification of conversation. }\end{array}$ & 10 \\
\hline $\begin{array}{l}\text { Communication with students- testing volume and understanding of speech } \\
\text { remotely }\end{array}$ & 2 \\
\hline Visuals - different screen sizes tested to identify ideal for patients. & 4 \\
\hline $\begin{array}{l}\text { Increased operational involvement for the patients, including writing, casting, } \\
\text { directing, and narrating. }\end{array}$ & 3 \\
\hline
\end{tabular}

\section{Abstract 7 Figure 1}

